\title{
Development and characterization of 22 polymorphic microsatellite markers for the balloon flower Platycodon grandiflorum (Campanulaceae)
}

\author{
J.Y. Song, G.-A. Lee, M.-S. Yoon, K.-H. Ma, Y.-M. Choi, J.-R. Lee, \\ H.-J. Park and M.-C. Lee
}

National Agrobiodiversity Center, National Academy of Agricultural Science, Rural Development Administration, Suwon, South Korea

Corresponding author: M.-C. Lee

E-mail: mcleekor@korea.kr

Genet. Mol. Res. 11 (3): 3263-3266 (2012)

Received November 8, 2011

Accepted June 19, 2012

Published September 12, 2012

DOI http://dx.doi.org/10.4238/2012.September.12.9

\begin{abstract}
The balloon flower (Platycodon grandiflorum A. DC.) is a perennial flowering plant of the Campanulaceae family; it is the only member of the genus Platycodon. Information on the genetic diversity of balloon flower populations is of great importance for the conservation and germplasm utilization of this flowering plant. Twenty-two polymorphic microsatellite loci were developed and characterized with eight balloon flower accessions collected from South Korea and China. Eighty-one alleles were detected among the eight balloon flower accessions. The number of alleles per locus ranged from two to six, with a mean of four alleles per locus. The observed and expected heterozygosity values ranged from 0.000 to 0.875 (mean $=0.355)$ and 0.117 to $0.766($ mean $=0.489)$, respectively. The polymorphic information content values ranged from 0.110 to 0.733 , with a mean of 0.449 . These new microsatellite markers will be useful for population and conservation genetic studies of P. grandiflorum.
\end{abstract}

Key words: Platycodon grandiflorum; Microsatellite markers; Genetic diversity; Conservation biology 


\section{INTRODUCTION}

Platycodon grandiflorum A. DC., known as bell flower or balloon flower, is the only species in the genus Platycodon L. of the family Campanulaceae. It is a herbaceous perennial native to northeastern Asia (Hong et al., 1983). Balloon flower is valued as a horticultural plant for its balloon-shaped flower. Its roots are popular in traditional Chinese medicine because they are abundant in triterpenoid saponins (Ishii et al., 1978; Fu et al., 2006) and used as a remedy for cough, cold, bronchitis, asthma, and sore throat (Germida et al., 1998; Lee et al., 2004). The root of balloon flower is commonly called doraji in South Korea, jie geng in China, and kikyo in Japan, and total saponin content varies among the germplasm resources (Kim et al., 2005).

Balloon flower is widely distributed in South Korea, but it is difficult to classify based on its morphological features aside from flower color and type. Furthermore, the root growth of domesticated balloon flower is faster than that of the wild type, and the root number of some landraces is higher than that in others. The aerial parts of $P$. grandiflorum are very similar to those of the wild type. Understanding the genetic variation, genetic structure, and phylogenetic characteristics of this species is important for its conservation and sustainable use, but molecular markers to classify genetic diversity in P. grandiflorum have not yet been reported.

Microsatellite markers are highly efficient molecular tools for studies on evolution, molecular ecology, and conservation biology owing to their high levels of polymorphism and high reproducibility. The present study is the first on the development and characterization of microsatellite markers in balloon flower.

\section{MATERIAL AND METHODS}

Microsatellite enrichment was performed according to the method of Kwon et al. (2009). Genomic DNA was extracted from the young leaves of $P$. grandiflorum using the Plant DNAzol reagent (Invitrogen) and was completely digested with 7 restriction enzymes: EcoRV, DraI, SmaI, $P v u \mathrm{I}, A l u \mathrm{I}$, HaeIII, and RsaI. After pooling, size-selected DNA (300-1500 bp) was isolated on agarose gel using a gel extraction kit (Qiagen). DNA fragments were then ligated to a blunt-end adaptor (AP11: 5'-CTC TTG CTT AGA TCT GGA CTA-3' and AP12: 5'-TAG TCC AGA TCT AAG CAA GAG CAC A-3') and hybridized with a mixture of biotin-labeled simple sequence repeat $(\mathrm{SSR})$ probes $\left[(\mathrm{GA})_{20},(\mathrm{CA})_{20},(\mathrm{AGC})_{15},(\mathrm{AAG})_{15},(\mathrm{AAC})_{15}\right.$, and $\left.(\mathrm{AGG})_{15}\right]$. The hybridized DNA fragments were subsequently captured on streptavidin-coated magnetic beads (Promega, USA), then cloned into vector PCR TOPO (Invitrogen) and transformed into chemically competent Escherichia coli cells (Invitrogen) for cloning. A total of 640 white colonies were randomly picked from the cloning plates. Plasmid DNAs were isolated using the QIAprep Spin Miniprep kit (Qiagen) and sequenced using an ABI 3100 DNA sequencer (Applied Biosystems, USA). SSR Manager (Kim, 2004) was used to identify the motifs within the cloned sequences and design primer pairs covering these regions.

The 640 clones were sequenced, and 43 clones $(6.7 \%)$ revealed redundancy. Four hundred and sixty-nine $(78.6 \%)$ of 597 unique clones contained microsatellite sequences, of which 281 clones had flanking regions suitable for primer design. The 281 pairs of primers were designed from flanking sequences of microsatellite motifs. Twenty-two of these microsatellites were successfully amplified and showed polymorphism in the 8 balloon flower accessions collected from South Korea and China. The M13-tail at the 5'-end region PCR method was used to measure 
the sizes of the amplified products (Schuelke, 2000). Amplified fluorescent-labeled PCR products were analyzed in an Abi-Prism 3130x1 Genetic Analyzer (Applied Biosystems). Fragments were sized and scored into alleles using GeneMapper v4.0 (Applied Biosystems).

The total number of alleles, expected and observed heterozygosities, and deviations from HWE for each SSR locus were calculated with PowerMarker version 3.25 (Liu and Muse, 2005).

\section{RESULTS AND DISCUSSION}

Eight-one alleles were detected, with an average of 4 alleles per microsatellite locus (Table 1).

\begin{tabular}{|c|c|c|c|c|c|c|c|c|c|c|}
\hline Primer No. & $\begin{array}{l}\text { GenBank } \\
\text { accession No. }\end{array}$ & Primer sequence (5'-3') & R-motif & $\begin{array}{l}\text { Size range } \\
\text { (bp) }\end{array}$ & $\mathrm{M}_{\mathrm{AF}}$ & $N_{\mathrm{A}}$ & $H_{\mathrm{E}}$ & $H_{\mathrm{O}}$ & PIC & HWE \\
\hline$\overline{\text { GB-PG-002 }}$ & JN712719 & $\begin{array}{l}\text { F-AAGCGGAAACCAAACTCC } \\
\text { R-GACCGCTCTCCATATCCC }\end{array}$ & $(\mathrm{CA})_{7} \ldots(\mathrm{GA})_{9}$ & $258-266$ & 0.625 & 4 & 0.563 & 0.000 & 0.524 & 0.000 \\
\hline GB-PG-004 & JN712720 & $\begin{array}{l}\text { F-TTTTCCAGAATCCTTCCTCA } \\
\text { R-GGCAAGTCTTGTAGGGAAAG }\end{array}$ & $(\mathrm{CT})_{15}$ & $213-237$ & 0.375 & 6 & 0.766 & 0.750 & 0.733 & 0.544 \\
\hline GB-PG-027 & JN712721 & $\begin{array}{l}\text { F-TCGATTCACCCGAAAATG } \\
\text { R-AACCACCAACCTACCCGT }\end{array}$ & $(\mathrm{GA})_{9}$ & $274-296$ & 0.688 & 4 & 0.484 & 0.500 & 0.443 & 0.552 \\
\hline GB-PG-029 & JN712722 & $\begin{array}{l}\text { F-CATCCAGTTGGTTTATCCACA } \\
\text { R-CTGCCCGTGAGAGAAGTG }\end{array}$ & $(\mathrm{CA})_{13}$ & 176-190 & 0.375 & 6 & 0.750 & 0.875 & 0.712 & 0.037 \\
\hline GB-PG-032 & JN712723 & $\begin{array}{l}\text { F-TTGTCTTCAACCCATCGC } \\
\text { R-TGCTTCCTTCGACACACC }\end{array}$ & $(\mathrm{TC})_{5} \ldots(\mathrm{CA})_{8}$ & 183-197 & 0.500 & 4 & 0.656 & 0.250 & 0.605 & 0.005 \\
\hline GB-PG-038 & JN712724 & $\begin{array}{l}\text { F-TAATAACCCCACCCCTGC } \\
\text { R-CGACGATCCGATTGGTTA }\end{array}$ & $(\mathrm{CA})_{8}$ & $195-203$ & 0.625 & 5 & 0.570 & 0.375 & 0.539 & 0.008 \\
\hline GB-PG-040 & JN712725 & $\begin{array}{l}\text { F-GCAATCTCTTGAGCGAACTT } \\
\text { R-TGGTTCAGCTTTGTCACG }\end{array}$ & $(\mathrm{GAA})_{4} \ldots(\mathrm{CA})_{10}$ & $310-320$ & 0.786 & 3 & 0.357 & 0.429 & 0.325 & 1.000 \\
\hline GB-PG-050 & JN712726 & $\begin{array}{l}\text { F-ACCGTTTGTTGTTGTCGC } \\
\text { R-TGTGAACCGACCCATTTC }\end{array}$ & $(\mathrm{GT})_{11}$ & $165-182$ & 0.571 & 5 & 0.622 & 0.714 & 0.587 & 0.712 \\
\hline GB-PG-059 & JN712727 & $\begin{array}{l}\text { F-CGACACAGCTACCAAATGC } \\
\text { R-CTGCTATGGTCAGTCGGC }\end{array}$ & $(\mathrm{GA})_{4}$ & $312-315$ & 0.857 & 2 & 0.245 & 0.286 & 0.215 & 1.000 \\
\hline GB-PG-069 & JN712728 & $\begin{array}{l}\text { F-TCCATTAAAAGGACCGCC } \\
\text { R-CCACCTCCTAAAGATGCCT }\end{array}$ & $(\mathrm{TTTA})_{4}$ & $318-327$ & 0.375 & 5 & 0.742 & 0.500 & 0.701 & 0.102 \\
\hline GB-PG-076 & JN712729 & $\begin{array}{l}\text { F-GATTTTCAACCGCCATCA } \\
\text { R-CGCTCAACAAAAAGGTCG }\end{array}$ & $(\mathrm{CT})_{17}$ & $232-252$ & 0.500 & 3 & 0.625 & 0.500 & 0.555 & 0.039 \\
\hline GB-PG-086 & JN712730 & $\begin{array}{l}\text { F-CGTCGCGTAGGACCTCTA } \\
\text { R-AGGGTCCCGCATCTTTTA }\end{array}$ & $(\mathrm{CCT})_{3} \mathrm{CTA}(\mathrm{CCT})_{3}$ & $268-316$ & 0.857 & 2 & 0.245 & 0.000 & 0.215 & 0.072 \\
\hline GB-PG-096 & JN712731 & $\begin{array}{l}\text { F-CATAGACAGCCACCGAGC } \\
\text { R-TTGCATCATCTTCTCCGTC }\end{array}$ & $(\mathrm{TC})_{3} \ldots(\mathrm{TC})_{3}$ & $244-252$ & 0.625 & 3 & 0.539 & 0.375 & 0.482 & 0.006 \\
\hline GB-PG-099 & JN712732 & $\begin{array}{l}\text { F-TTTATGCCTTGTGTTTGAAGC } \\
\text { R-AGGGAGATCGAGCCAAAA }\end{array}$ & $(\mathrm{GGA})_{5}$ & $249-252$ & 0.938 & 2 & 0.117 & 0.125 & 0.110 & 1.000 \\
\hline GB-PG-119 & JN712734 & $\begin{array}{l}\text { F-TTGGTGGATGCCGTTAGT } \\
\text { R-GGATTCGGGTTTCGAAGT }\end{array}$ & $(\mathrm{CA})_{8} \ldots(\mathrm{CA})_{16}$ & 342 & 0.625 & 4 & 0.555 & 0.125 & 0.510 & 0.001 \\
\hline GB-PG-127 & JN712735 & $\begin{array}{l}\text { F-AGCTCCCTTCTGCCTTTG } \\
\text { R-TCGCTGACTCTCTCCCTTT }\end{array}$ & $(\mathrm{TG})_{3} \ldots(\mathrm{TG})_{4}$ & $215-225$ & 0.833 & 2 & 0.278 & 0.000 & 0.239 & 0.100 \\
\hline GB-PG-128 & JN712736 & $\begin{array}{l}\text { F-CCCACCACTTTCCCTCTC } \\
\text { R-CGTTTGTGGAAAGAACGG }\end{array}$ & $(\mathrm{CAA})_{7}$ & 310-319 & 0.813 & 3 & 0.320 & 0.125 & 0.294 & 0.072 \\
\hline GB-PG-149 & JN712737 & $\begin{array}{l}\text { F-GGCGATTTGAGAGGCATA } \\
\text { R-AGACCCGTTCCATAGAAAGTT }\end{array}$ & $(\mathrm{CCCACT})_{3} \ldots(\mathrm{CA})_{5}$ & 289-331 & 0.563 & 5 & 0.625 & 0.625 & 0.586 & 0.378 \\
\hline GB-PG-171 & JN712738 & $\begin{array}{l}\text { F-GTCGCTTGGATGTGATGC } \\
\text { R-GGTTCAAAACGCAACTTCC }\end{array}$ & $(\mathrm{CA})_{13}$ & 194-202 & 0.500 & 4 & 0.602 & 0.375 & 0.525 & 0.033 \\
\hline GB-PG-177 & JN712739 & $\begin{array}{l}\text { F-CCCAATGAGCAAATCAGC } \\
\text { R-GCCTATATTCCCGTTGCC }\end{array}$ & $(\mathrm{TG})_{9}$ & $154-176$ & 0.813 & 4 & 0.328 & 0.375 & 0.313 & 1.000 \\
\hline GB-PG-182 & JN712740 & $\begin{array}{l}\text { F-AAAGACCGACCCAGAAGC } \\
\text { R-TATGCACGCAAAATTCCC }\end{array}$ & $(\mathrm{GAA})_{4}$ & $170-182$ & 0.750 & 3 & 0.398 & 0.250 & 0.354 & 0.382 \\
\hline GB-PG-197 & JN712742 & $\begin{array}{l}\text { F-AGTCTGCCGTCCCATTCT } \\
\text { R-TCCCTGCACAAACAAACA }\end{array}$ & $(\mathrm{CA})_{9}$ & $266-268$ & 0.750 & 2 & 0.375 & 0.250 & 0.305 & 0.405 \\
\hline & - & - & - & - & & 81 & & & & ro \\
\hline Mean & - & . & 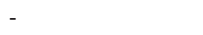 & . & 0.652 & 4 & 0.489 & 0.355 & 0.449 & 0.339 \\
\hline
\end{tabular}

$\mathrm{M}_{\mathrm{AF}}=$ major allele frequency; $N_{\mathrm{A}}=$ number of alleles; $H_{\mathrm{E}}$ and $H_{\mathrm{O}}=$ expected and observed heterozygosities; PIC = polymorphic information content; HWE $=$ Hardy-Weinberg equilibrium. 
Of these markers, only 2 (GB-PG-004 and GB-PG-029) produced the highest number of alleles (6), whereas 5 markers (GB-PG-059, GB-PG-086, GB-PG-099, GB-PG-127, and GB-PG-197) detected just 2 alleles. The observed heterozygosity ranged from 0.000 to 0.875 $($ mean $=0.355)$, and the expected heterozygosity from 0.117 to $0.766($ mean $=0.489)$. The polymorphic information content ranged from 0.110 to 0.733 , with an average of 0.449 (Table 1). The 22 microsatellite markers developed will be useful for future genetic diversity, classification, and genetic structure studies in P. grandiflorum.

\section{ACKNOWLEDGMENTS}

Research supported by the Research Program for Agricultural Science and Technology Development (\#PJ008368) and the 2012 Post-Doctoral Fellowship Program of the National Academy of Agricultural Science, Rural Development Administration, Republic of Korea.

\section{REFERENCES}

Fu WW, Shimizu N, Dou DQ, Takeda T, et al. (2006). Five new triterpenoid saponins from the roots of Platycodon grandiflorum. Chem. Pharm. Bull. 54: 557-560.

Germida JJ, Siciliano SD, Freitas JR and Seib AM (1998). Diversity of root-associated bacteria associated with fieldgrown canola (Brassica napus L.) and wheat (Triticum aestivum L.). FEMS Microbiol. Ecol. 26: 43-50.

Hong DY, Lian YS and Shen LD (1983). Flora Reipublicae popularis sinicae. Sci. Press. 73: 76-77.

Ishii H, Tori K, Tozyo T and Yoshimura Y (1978). Structures of polygalacin-D and -D2, platycodin-D and -D2, and their monoacetates, saponins isolated from Platycodon grandiflorum A. DC., determined by carbon-13 nuclear magnetic resonance spectroscopy. Chem. Pharm. Bull. 26: 674-677.

Kim KY (2004). Developing a One Step Program (SSR Manager) for Rapid Identification of Clones With SSRs and Primer Design. Master's thesis, Seoul National University, Seoul.

Kim YS, Kim JS, Choi SU, Kim JS, et al. (2005). Isolation of a new saponin and cytotoxic effect of saponins from the root of Platycodon grandiflorum on human tumor cell lines. Planta Med. 71: 566-568.

Kwon S-J, Lee G-A, Lee S-Y, Park Y-J, et al. (2009). Isolation and characterization of 21 microsatellite loci in Lycium chinense and cross-amplification in Lycium barbarum. Conserv. Genet. 10: 1557-1560.

Lee JY, Hwang WI and Lim ST (2004). Antioxidant and anticancer activities of organic extracts from Platycodon grandiflorum A. De Candolle roots. J. Ethnopharmacol. 93: 409-415.

Liu K and Muse SV (2005). PowerMarker: An Integrated Analysis Environment for Genetic Marker Analysis. Oxford University Press, Oxford.

Schuelke M (2000). An economic method for the fluorescent labeling of PCR fragments. Nat. Biotechnol. 18: 233-234. 